\title{
Position and density effects on repression by stationary and mobile DNA-binding proteins
}

\author{
Stephen J. Elledge and Ronald W. Davis \\ Department of Biochemistry, Stanford University School of Medicine, Stanford, California 94305 USA
}

\begin{abstract}
We have investigated the effects of two types of DNA-binding proteins on bacterial repression. First, the effects of operator positioning on repression by stationary DNA-binding proteins, the Lac repressor and the Trp repressor, were examined in vivo. Both operator number and positioning play a role in determining in vivo levels of repression. Operators located within a promoter are more efficient regulators than those positioned at the start of transcription. Second, we investigated the effects of DNA-binding protein density on repression using a mobile DNA-binding protein, Escherichia coli RNA polymerase. We employed a transcriptional interference assay using convergent transcriptional units. The strong synthetic promoter conI and its derivatives were observed to interfere with expression of the aadA gene, which confers spectinomycin resistance upon its host. Transcriptional interference by RNA polymerase occurred only in cis and had a strong dependence on polymerase density that was modulated by varying the promoter strengths. A change in the density of approximately fourfold completely abolished the observed transcriptional interference. Several models are discussed to explain the repression patterns observed for stationary and mobile DNA-binding proteins.
\end{abstract}

[Key Words: Lac repressor; Trp repressor; trp promoter; operating positioning; transcriptional interference; genetic selection]

Received October 11, 1988; revised version accepted December 16, 1988.

The accurate and efficient regulation of gene expression is central to an organism's ability to undergo complex developmental transformations and to adapt successfully to changing environmental conditions. Consequently, organisms have evolved a rich and varied repertoire of mechanisms to control gene expression. These include the ability to modulate the activity of gene expression at every conceivable step in the cascade of expression from the abundance, accessibility, and structural continuity of DNA to the most subtle nuances of catalytic activity and structural integrity of proteins.

The step at which control is most often observed is the level of initiation of transcription. This is true for two reasons. First, it is the most energy-efficient control mechanism for the organism, circumventing the energy cost of transcription, translation, processing, and catabolism; second, it is the simplest type of regulation to detect experimentally and therefore is searched for most frequently and explored most thoroughly. Regulation of transcription is divided into two basic types, positive and negative, classically defined by whether the absence of a functional gene known to provide regulation causes an increase or a decrease in the rate of transcriptional initiation. More recently, this distinction has become blurred with the more detailed inspections of systems of greater and greater complexity.

Negative regulation was first elucidated by Jacob and
Monod for the lacZYA operon of Escherichia coli. Although more and more complex examples of negative regulation are being discovered, we still lack a thorough basic understanding of precisely how the simplest systems function. For instance, the effect of operator number and position on the initiation of transcription remains largely unexplored. The initiation of transcription is thought to proceed in three steps /Chamberlin 1974; Buc and McClure 1985). First, RNA polymerase binds to the promoter to form the 'closed complex,' $\mathrm{RP}_{\mathrm{c}}$. Then the DNA near the start of transcription is denatured, thus defining the formation of the more stable 'open complex,' RP (Kirkegaard et al. 1983). Upon addition of ribonucleotides, the 'initiation complex' forms, $\mathrm{RP}_{\mathrm{i}}$ may actually constitute two steps; the formation of a recycling abortive initiation complex and the subsequent conversion to a stable elongation complex that has been correlated with the loss of the $\sigma$-subunit after transcription of 9-11 nucleotides (Carpousis and Gralla 1980; Hansen and McClure 1980). Abortive cycling has yet to be demonstrated in vivo and may be an in vivo artifact. Although most models of repressor action suggest inhibition of polymerase binding as the mechanism of repression (Majors 1975; Squires et al. 1979; Ptashne et al. 1980), there is no reason to think that a repressor could not act by inhibiting any one of the initiation steps mentioned above. In fact, Schmitz and Galas 
(1979) have shown that binding of the Lac repressor does not preclude binding of RNA polymerase. Furthermore, Straney and Crothers (1987) have shown that the Lac repressor can actually increase the initial binding of RNA polymerase and that it represses by inhibiting the formation of the open complex when bound at +1 . To address these questions concerning the mode of negative regulation proposed for the lac and trp operons, we performed a detailed analysis of operator-promoter interaction to determine how distance and geometry affect negative regulation.

Recently, much attention has been directed toward the question of how proteins can exert their influence at a distance. This type of regulation is ubiquitous in nature and varied in mechanism. It is involved in dosage compensation in mammals, silencer function in fungi (Brand et al. 1985), and archival DNA in bacteria (Downs and Roth 1987). These are examples of negative regulation that function over long distances, as opposed to the elegant and seemingly simple local mode of negative regulation proposed for the lac operon. Three general classes of mechanisms have been described for such distal actions (Wang and Giaever 1988): tracking of a protein along a DNA, the association of multiple proteins at separate sites to form a DNA loop in between, and distal interactions that are affected by the topology of the DNA. In the second part of our analysis of negative regulation, we examine the role that translocation of RNA polymerase along the DNA plays in repression. We demonstrate that complex regulatory circuitry can be assembled out of simple fundamental regulatory units. These new combinatorial units can now function at a distance and are able to amplify small changes in occupancy into much larger effects.

\section{Results}

The Lac repressor shows decreased repression with increasing distance

To determine the precise relationship between promoter function and operator position, a well-characterized promoter lacking all regulation was needed. Because few promoters fall into this category, an existing promoter, tac, was modified to remove its regulatory sequences. $t a c$ is a fusion promoter containing the -35 region of the trp promoter and the -10 region of the lac promoter. This hybrid promoter removes the trpR regulatory sequences from the trp promoter and the catabolite-repressing sequences from the lac promoter but leaves the lac operator sequences intact (Amann et al. 1983). Oligonucleotide site-directed mutagenesis was used to remove the lac operator from tac to create a strong constitutive promoter conI (constitutive) (Fig. 1) as detailed in Materials and methods. An EcoRI restriction site was placed at the start of transcription, followed by a polylinker to facilitate operator positioning (Fig. 2A). We constructed new regulated promoters with the 20-bp lac operator spaced approximately every $2 \mathrm{bp}$ from +5 to +40 , relative to the start of transcription. The sequence
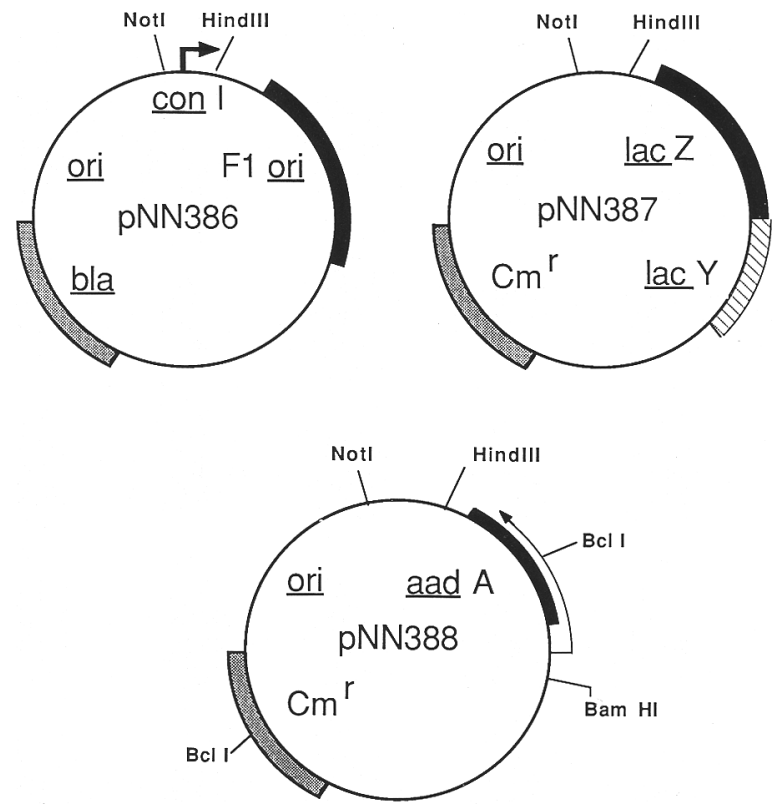

Figure 1. Maps of plasmids used in this study. pNN386 is a derivative of pEMBL18, as described in Materials and methods. It contains the conI promoter and polylinker, the sequence of which is illustrated in Fig. 2. This plasmid was used as the base vector for the creation of all of the Lac repressor-regulated promoters shown in Fig. 2. pNN396 is a derivative of pNN386 and has the conII promoter (Fig. 3) in place of conI. pNN387 is a single-copy-number plasmid used to measure the promoter strength of the regulated promoters used in this study. The LacZY genes on pNN387 lack a promoter. pNN388 is a singlecopy-number plasmid used to measure the ability of transcription to interfere with the expression of the aadA gene. The arrows in this diagram represent the direction of transcription of their respective genes. These maps are not drawn to scale.

of each construct is listed in Figure 2B. These promoter constructs were assayed by allowing them to direct $\beta$ galactosidase synthesis in isogenic lacI- or lacIq backgrounds, as described in Materials and methods /the lacI ${ }^{q}$ mutation results in a 10 -fold increase in LacI synthesis). The results of the assays are shown in Figure 2B. Because the precise sequence of the 5' end of each message varies slightly, we cannot strictly control for message stability. Therefore, repressed and induced levels cannot be directly compared from one construct to the next. However, the induction ratio should control for the message stability and can be directly compared if we assume that the structures of the mRNA for the repressed and induced states are the same. The induction ratios for the modified conI promoters are listed in Figure 2B. The repression appears to show a tripartite pattern. Operators positioned between +5 and +14 give high levels of repression, $\sim 500$-fold. However, positions +17 to +31 give a consistently lower induction ratio of $\sim 100$-fold. Finally, induction ratios begin to drop after +30 , giving a final ratio of 29 -fold at +40 . Several points should be emphasized. First, con promoters lacking the synthetic lacO oligonucleotide still show a twofold in- 
A

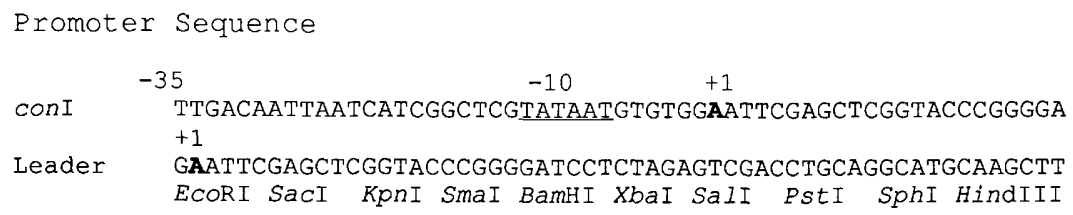

B

lac Operator Insertions

\section{Position Sequence}

$I-1(+5)$

$I-2(+7)$

$I-3(+9)$

$I-4(+11)$

$I-5(+13)$

$I-6(+14)$

$I-7(+17 A)$

$\mathrm{I}-8(+17 \mathrm{~B})$

$I-9(+19)$

$I-10(+21)$

$I-11(+23)$

$I-12(+25)$

$I-13(+27)$

$I-14(+31)$

$I-15(+34)$

$I-16(+40)$

conI

\begin{abstract}
GAATTAATTGTGAGCGCTCACAATTAATTCGAGCTCGGTACCCGGGGATCCTCTAGAGTC GAATTCGAATTGTGAGCGCTCACAATTCGGTACCCGGGGATCCTCTAGAGTCGACCTGCA GAATTCGCGAATTGTGAGCGCTCACAATTCCGGGGATCCTCTAGAGTCGACCTGCAGGCA GAATTAATTCGAATTGTGAGCGCTCACAATTCGGTACCCGGGGATCCTCTAAGAGTCGAC GAATTCGAGCTCGAATTGTGAGCGCTCACAATICCGGGGATCCTCTAGAGTCGACCTGCA GAATTCGGGTACCCAATTGTGAGCGCTCACAATTGGGGATCCTCTAGAGTCGACCTGCAG GAATTCGAGCTCGGTACAATTGTGAGCGCTCACAATTGTACCCGGGGATCCTCTAGAGTC GAATTCGCGGTACCCGGAATTGTGAGCGCTCACAATTCCGGGGATCCTCTAGAGTCGACC GAATTCGAGCTCGGTACCCAATTGTGAGCGCTCACAATTGGGGATCCTCTAGAGTCGACC GAATTCGAGCTCGGTACCCGGAATTGTGAGCGCTCACAATTCCGGGGATCCTCTAGAGTC GAATTAATTCGAGCTCGGTACCCAATTGTGAGCGCTCACAATTGGGGATCCTCTAGAGTC GAATTAATTCGAGCTCGGTACCCGGAATIGTGAGCGCTCACAATTCCGGGGATCCTCTAG GAATTCGCGGTACCCGGGGATCCTCTAAATTGTGAGCGCTCACAATTAGAGTCGACCTGC GAATTCGAGCTCGGTACCCGGGGATCCTCTAAATTGTGAGCGCTCACAATTTAGAGTCGA GAATAATTCGAGCTCGGATCCCGGGGATCCTCTAAATTGTGAGCGCTCACAATTTAGAGT GAATTCGAGCTCGGTACCCGGGGATCCTCTAGAGTCGACCAATTGTGAGCGCTCACAATT GAATTCGAGCTCGGTACCCGGGGATCCTCTAGAGTCGACCTGCAGGCATGCAAGCTT . . .
\end{abstract}

$\begin{array}{lll}\begin{array}{l}\text { B-galactosidase } \\ \text { units }\end{array} & \\ \text { lacI }^{9} & \text { lacI }^{-} & \text {Ratio } \\ 0.61 & 270 & 442 \\ 0.40 & 357 & 893 \\ 0.64 & 324 & 506 \\ 0.80 & 348 & 420 \\ 0.60 & 310 & 517 \\ 0.82 & 206 & 251 \\ 9.3 & 478 & 51 \\ 3.2 & 363 & 113 \\ 1.5 & 303 & 202 \\ 1.8 & 209 & 116 \\ 2.1 & 214 & 102 \\ 2.1 & 210 & 100 \\ 1.8 & 212 & 117 \\ 2.7 & 270 & 107 \\ 3.2 & 239 & 75 \\ 8.2 & 237 & 29 \\ 163 & 346 & 2\end{array}$

Figure 2. The construction of lacI-regulated promoters. $(A)$ This shows the sequence of the constitutive promoter conI, the construction of which is outlined in Materials and methods. The base at the start of transcription is indicated in boldface type. The sequence of the polylinker leader region is shown, along with the various restriction enzymes that recognize it. $(B)$ The sequence of the con $I$ - 1 through conI-16 lacI-regulated promoters. Their construction is detailed in Materials and methods. The start of transcription is indicated in boldface type. The positions of the bases contributed by the 20-bp synthetic lac operator are underlined in each promoter.

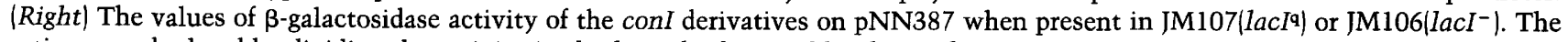
ratio was calculated by dividing the activity in the 1 acI- background by that in lacIq.

duction. This is probably due to the presence of a lacO sequence within the $l a c Z$ gene. When the repressor is bound at this site, it can act as a transcription terminator and thereby partially repress the production of a full-length functional message (Deuschle et al. 1988a). The lac operator within the lacZ gene of pNN387 is 1.3 $\mathrm{kb}$ from the start of transcription and whatever contribution it makes to repression should remain constant among the various constructs. Induction ratios listed here should be divided by 2 when considered in absolute terms. The true normalizing factor may be slightly larger if there is an interaction between operators, as suggested by Mossing and Record (1986). Second, it should be noted that the largest contribution to the change in induction ratio can be attributed to an increase in the level of expression in the repressed state because the levels in the absence of repressor are approximately equal for most constructs. Third, a sequence context effect on repressor function exists. Two different constructs, both bearing an operator at position +17 but in a different sequence context, give a difference of twofold in the induction ratio. Therefore, not only is the po- sition of the operator important in determining repressor effectiveness, but its precise context must also play a role. This may be due to an altered operator affinity or to the complex rules governing the interplay of polymerase and repressor that determine the repressed levels of expression. For example, if the polymerase enhances the 'off' rate of the repressor by unwinding the DNA, then repressors whose operators are imbedded in GC-rich sequences may be slightly more efficient repressors than those imbedded in AT-rich sequences (cf. conI-7 and conI-8).

\section{Operator position is more critical for Trp repressor function}

The Lac repressor is an exceptional molecule. Its dissociation constant has been estimated to be $10^{-13} \mathrm{M}$ (Riggs et al. 1969). This tight binding is thought to be responsible for its ability to terminate transcription. It is possible that other repressor molecules with somewhat less extreme properties might not share the pattern of repression observed for the Lac repressor. As a second model, we chose the Trp repressor encoded by $\operatorname{trp} R$. The 
Trp repressor was chosen because of its smaller size (24$\mathrm{kD}$ dimer as opposed to a $150-\mathrm{kD}$ tetramer) and its weaker dissociation constant $2 \times 10^{-9} \mathrm{M}$ (Klig et al. 1987; Carey 1988).

To perform a systematic analysis of Trp repression, a second constitutive promoter, conII, was constructed. The sequence of conII is shown in Figure $3 \mathrm{~A}$. conII is a derivative of conI that has a $K p n I$ site at -6 relative to the start of transcription, allowing a greater range of operator positioning. The trp operator sequence chosen for analysis is illustrated in Figure $3 B$ and was taken from the central region of the trp promoter $(-19$ to -1$)$. This region has been shown to footprint with the Trp repressor in vitro and is the region to which operator constitutive mutations map in the trp promoter (Bennett and Yanofsky 1978). New promoters were created with the synthetic trp operator spaced $\sim 3-4$ bp apart from -5 to +16 relative to the start of transcription. The se- quence of each construct is shown in Figure 3B. These promoter constructs were assayed by allowing them to direct $\beta$-galactosidase synthesis on pNN387 in isogenic $\operatorname{trp} R^{+}$and $\operatorname{trp} R^{-}$backgrounds, as described in Materials and methods. The induction ratios for the modified conII promoters are shown in Table 1 . The ability to repress shows a dramatic distance effect, dropping to barely detectable levels between +9 and +16 . There also appear to be slight positioning effects; operators placed at -5 repress at only $50 \%$ of the levels of those at positions $-1,+2$, and +5 , suggesting that the precise geometrical relationship between repressor and polymerase may play a role in repression among operators located within a given area. Of course, sequence contest effects as observed for the Lac repressor cannot be ruled out. In general, operator position is more critical for the Trp repressor function and the levels of repression differ significantly from those obtained with the Lac repressor.

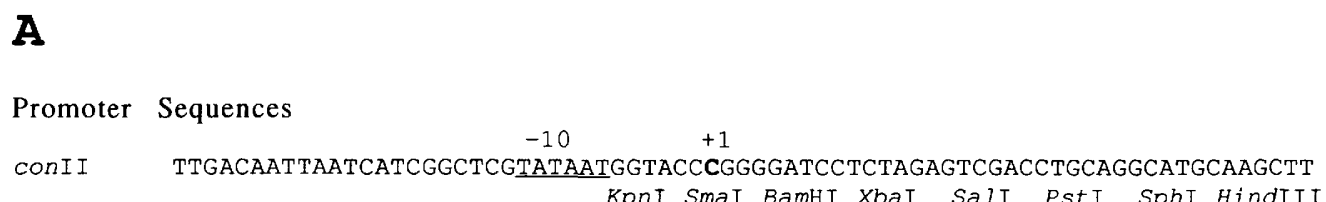

B

trp Operator Sequence $\underset{t r p O}{\operatorname{AACTAGTTAACTAGTACGCTGCAG}}$

trp Operator Insertions

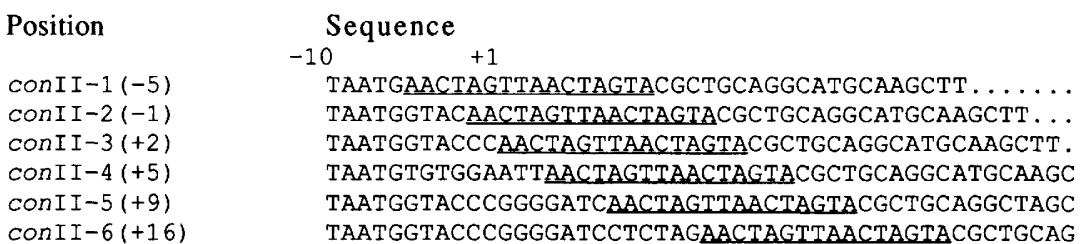

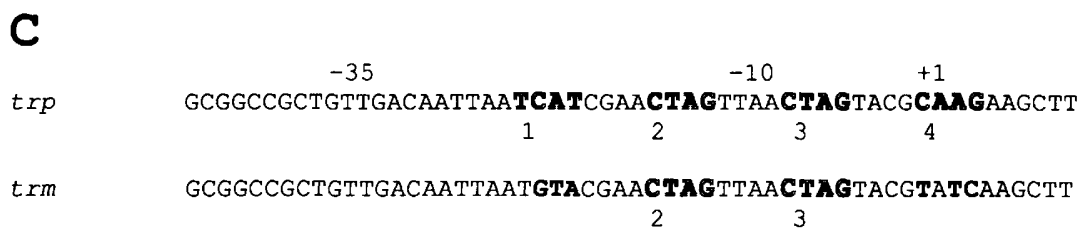

Figure 3. The construction of $t r p R$-regulated promoters. $(A)$ The sequence of the conII promoter present on pNN396. conII is a derivative of conI, and its construction is described in Materials and methods. The start of transcription is indicated in boldface type. (B) (Top line) The sequence of the synthetic trp operator oligonucleotide used to construct the trpR-regulated promoters. $1-19$ to -2 from $t r p \mid$ Beneath are illustrated the sequences of the conII-1 through conII-6 trpR-regulated promoters. The construction of these promoters is detailed in Materials and methods. The position of the trp operator is underlined in each promoter. $(C)$ The sequence of the trp promoter. The bases important for Trp repressor binding are outlined (Bass et al. 1987). Each set of four bases represents the essential elements of one half-site, and each half-site is numbered below. Kumamoto et al. (1987) suggest that three Trp repressor dimers bind the $t r p$ promoter. The first dimer binding half-sites 1 and 2 , the second binding 2 and 3 , and the third binding 3 and 4 . The Trp repressor can share half-sites because the critical bases within a half-site are pallindromic. The sequence of trm, a mutant form of trp, is shown below that of trp. It differs from trp at 6 bases, 3 each in half-sites 1 and 4. The mutational changes are indicated in boldface type. 
Table 1. Transcriptional activities of trpR-regulated promoters

\begin{tabular}{|c|c|c|c|c|c|}
\hline \multirow[b]{2}{*}{ Promoter } & \multicolumn{3}{|c|}{$\beta$-Galactosidase units } & \multicolumn{2}{|c|}{ Ratios } \\
\hline & $\overline{\operatorname{trp} R^{+}}$ & $\operatorname{trpR^{-}}$ & $\overline{\text { pRPG9a }^{a}}$ & $\overline{\left(R-/ R^{+}\right)}$ & (R-/pRPG9) \\
\hline $\operatorname{trp}$ & 6.5 & 792 & - & 120 & 一 \\
\hline $\operatorname{trm}(-19)$ & 22.5 & 779 & - & 34 & - \\
\hline $\operatorname{con} I I-1(-5)$ & 226 & 1382 & 115 & 6 & 12.0 \\
\hline $\operatorname{con} I I-2(-1)$ & 117 & 1266 & - & 11 & - \\
\hline $\operatorname{con} I I-3(+2)$ & 120 & 1755 & 93 & 14 & 18.8 \\
\hline $\operatorname{con} I I-4(+5)$ & 63 & 637 & - & 10 & - \\
\hline conII-5|+9| & 375 & 1022 & 266 & 3 & 3.8 \\
\hline conII- $6|+16|$ & 122 & 273 & - & 2 & - \\
\hline conII & 720 & 605 & - & 0.9 & - \\
\hline
\end{tabular}

$\beta$-Galactosidase activities were measured for the promoters listed in Fig. 3 when placed onto pNN387. Activities are calculated according to Miller (1972) and are the average of at least two independent measurements. Activities typically varied no more than $15 \%$ between independent measurements.

${ }^{a}$ pRPG9 represents activities in a $\operatorname{trp} R^{+}$strain carrying pRPG9, a pBR322-based clone of $\operatorname{trp} R$, which overproduces the Trp repressor.

Repression of the trp promoter is determined by the number and positioning of operators

An outstanding question in prokaryotic regulation is why the $\operatorname{trp}$ promoter is so efficiently repressed relative to $\operatorname{trp} R$. The $\operatorname{Trp}$ repressor regulates three known loci, $\operatorname{trpEDCBA}$, $\operatorname{aroH}$, and $\operatorname{trpR}$. These operons show differing degrees of regulation in vivo. The $a r o H$ and $\operatorname{trp} R$ operons are regulated over a four- to fivefold range (Kelley and Yanofsky 1982; Grove and Gunslaus 1987), whereas the trp promoter is regulated over a 70 -fold range, excluding attenuation (Kelley and Yanofsky 1982). Evidence has been presented to suggest that these promoters vary in both the number and positioning of Trp repressor-binding sites (Kumamoto et al. 1987). These results suggest that the trp promoter may have three tandem repressor-binding sites covering $\sim 33$ bases $(-25$ to +7$)$ when footprinted at high concentrations of Trp repressor in vitro. Furthermore, their results suggest that $a r o H$ promoter has two tandem binding sites covering $\sim 30$ bases $(-50$ to -20$)$ and the trpR promoter has only one binding site covering 25 bases $(-10$ to +15$)$ when footprinted in vitro. Kumamoto et al. (1987) suggest that the difference in levels of repression is due to the fact that the trp promoter has three binding sites for the Trp repressor, whereas $\operatorname{trpR}$ has only one. So far, only the central operator in trp has been shown to be functional in vivo (Bennett and Yanofsky 1978). Alternatively, the differing positions of the operators within these promoters may determine the efficiency of repression. Differential operator affinities could also be involved. To test these hypotheses and to determine the effect of a single repressor binding site located in the central portion of the promoter recognition elements, a mutant trp promoter trm (trp mutant), was constructed that left the interior Trp repressor-binding site intact $(-19$ to -1$)$, but destroyed the outer two binding sites. The sequences of the trp and trm promoters are shown in Figure 3C. The mutations used to create trm are shown in boldface type. These changes were designed to combine all of the most severe operator-constitutive mutations for the trp operator, as determined by Bennett and Yanofsky (1978) and Bass et al. (1987) into each of the two exterior half-sites ( 1 and 4, Fig. 3B). The repression data for these promoters are in Table 1. The trp promoter is repressed 120-fold, whereas trm is repressed only 34 -fold. This evidence suggests that at least one of the other two trp operator sites function in vivo but that the majority of the repression is due to the central operator. Furthermore, it shows that operators positioned deep within the promoter element, overlapping critical RNA polymerase recognition sequences, are more efficient at repression than operators positioned at the start of transcription (cf. trm and -5 or -1 from Table 1). These data also suggest that both positioning and operator number play a role in determining the level of trp repression in vivo.

The repression data for trm suggest that a single trp operator has a minimal occupancy of $97 \%$ in vivo in the presence of the normal intracellular concentration of Trp repressor (assuming 99\% occupancy would be equivalent to 100 -fold repression). However, the same operator sequence located at a different position in the same promoter, e.g., -5 or -1 , has severalfold weaker repression. Does this mean that they have a significantly lower occupancy than the trm operator or that they have reached the maximal repressing potential at near maximal occupancy for operators located at those positions? One possible model is that when an operator is occupied near the start of transcription, a polymerase is also bound and sits poised, ready to transcribe when the repressor dissociates. In this model, the basal level of the promoter is determined solely by the off rate of the repressor at near full occupancy. This hypothesis would predict that under these conditions, increasing the 'on' rate of the repressor by increasing its concentration should have little effect upon the basal level of tran- 
scription. With this in mind, an experiment was performed, the results of which are listed in the last two columns of Table 1. pRPG9, a clone of the trpR gene on pBR322, was introduced into strains bearing several of the modified conII promoters. We estimate that pRPG9 overproduces the Trp repressor approximately fivefold. The data from this experiment indicate that increasing the concentration of repressor can increase repression $(30-100 \%)$ of operators located near the start of transcription significantly. This implies that the simple model as stated above is incorrect. One obvious possibility is that once the repressor falls off, it still has an opportunity to rebind before the polymerase initiates transcription. Two other interesting but more complex models are explored in the Discussion.

\section{Repression at a distance may be exerted by convergent transcription}

Up to this point, this work and other studies have focused primarily on forms of regulation involving stationary regulatory proteins that exert their influence over very short distances (tens of base pairs). However, the role played by mobile DNA-binding proteins to provide regulation that functions over longer distances $(\mathrm{ki}-$ lobases) has remained relatively unexplored. One mechanism for repression at a distance could be convergent transcription. To study this phenomenon, we designed the system illustrated in Figure 4A. The conI promoter was placed downstream from the aadA gene in an orientation such that it would transcribe aadA in an antisense direction to determine whether conI could affect aadA expression. aadA laminoglycoside adenyltransferase) encodes an enzyme that adenylates aminoglycoside antibiotics such as streptinomycin and spectinomycin and renders them ineffective (Hollisgshead and Vapnek 1984), thus providing resistance for the bacteria bearing this gene. Furthermore, there is some evidence that the adenylated amnioglycosides participate in increasing resistance by blocking transport of the unmodified aminoglycosides to the cell.

The conI promoter is able to interfere with expression of $\operatorname{aadA}$, a weakly expressed gene, and this effect can be monitored by measuring resistance to spectinomycin, as seen in Table 2. Strains containing pNN388 (Fig. 1), which lacks conI, plate with equal efficiency on media containing chloramphenicol or spectinomycin. However, strains bearing pNN389 (Fig. 4B), which contains conI opposing the aadA gene, plate efficiently on media containing chloramphenicol but are extremely sensitive to spectinomycin (i.e., appear aadA-). To determine whether this effect was due to transcription by conI and not merely the alteration of sequence $3^{\prime}$ to the aadA structural gene, a derivative of conI was used that had a lac operator placed at position +5 relative to the start of transcription (Fig. 2). As shown in Table 2, insertion of a lac operator (pNN394) restored function to the aadA gene in a $\mathrm{lacI}^{+}$background but that this function was again interrupted upon induction of antisense transcription by the addition of isopropyl- $\beta$ - $D$-thiogalactoside
A
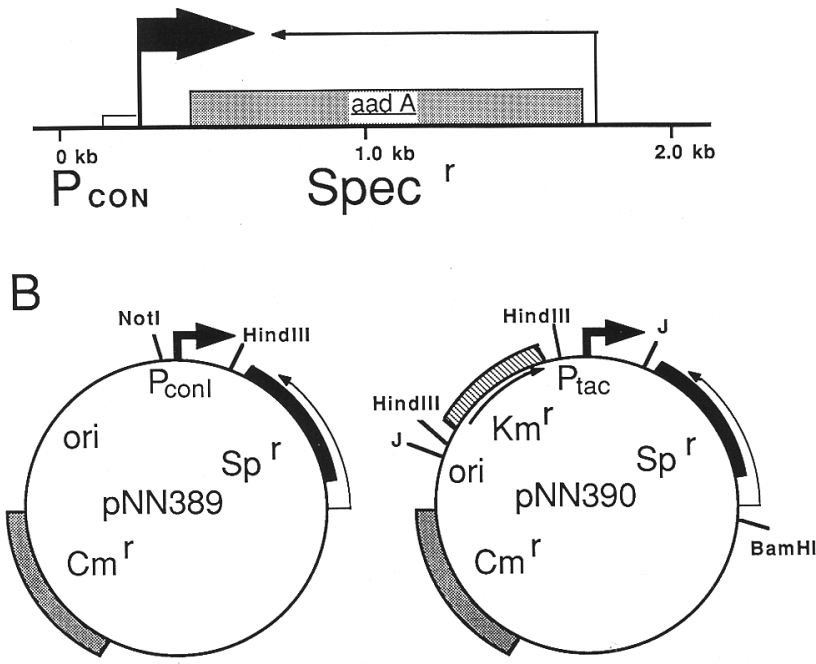

Figure 4. Schematic representations of promoter arrangements producing transcriptional interference. $|A|$ A map of the relative positions of the con promoter derivatives and aadA. Arrows indicate the direction of transcription and relative promoter strength. $(B)$ Restriction maps of pNN389 and pNN390 (Table 3). Restriction sites relevant to their construction are shown. (J) A junction created during the cloning in which the restriction sites were destroyed. These maps are not shown to scale.

(IPTG). The presence of the lac operator had no effect in a lacI- background nor did IPTG. Therefore, we conclude that the induced sensitivity phenotype is due to transcription.

Several different mechanisms could explain this ap-

Table 2. cis and trans effects on aadA expression

\begin{tabular}{|c|c|c|c|}
\hline \multirow[b]{3}{*}{ Plasmid(s) } & \multirow{3}{*}{$\begin{array}{l}\text { lacI } \\
\text { genotype }\end{array}$} & \multirow{2}{*}{\multicolumn{2}{|c|}{$\begin{array}{c}\begin{array}{c}\text { Efficiency of plating } \\
\text { on spectinomycin }\end{array} \\
\text { IPTG }\end{array}$}} \\
\hline & & & \\
\hline & & $1-1$ & $1+1$ \\
\hline pNN388 & $q$ & 1 & 1 \\
\hline pNN389(conI) & $q$ & $10^{-6}$ & $10^{-6}$ \\
\hline pNN394(conI-1) & $\mathrm{q}$ & 1 & $10^{-6}$ \\
\hline pNN394 & - & $10^{-6}$ & $10^{-6}$ \\
\hline pNN395(conI-16) & $q$ & 1 & $10^{-6}$ \\
\hline pNN395 & - & $10^{-6}$ & $10^{-6}$ \\
\hline pNN390(tac $\left.{ }^{+}\right)$ & $q$ & 1 & $10^{-6}$ \\
\hline pNN391 $\left(\right.$ tac $\left.^{-}\right)$ & $\mathrm{q}$ & 1 & 1 \\
\hline pRMM61 & $q$ & 1 & 1 \\
\hline pNN390, pRMM61 & $\mathrm{q}$ & 1 & 1 \\
\hline pNN392 & $\mathrm{q}$ & 1 & $10^{-4}$ \\
\hline pNN393 & $\mathrm{q}$ & $<10^{-7}$ & $<10^{-7}$ \\
\hline pNN388, pNN393 & $\mathrm{q}$ & 1 & 1 \\
\hline
\end{tabular}

Dilutions of cells were plated on LB plates supplemented with spectinomycin $(80 \mu \mathrm{g} / \mathrm{ml})$, with or without $3 \mathrm{mM}$ IPTG. With respect to the lacI genotype, q represents $l a c I^{q}$ and - represents $1 a c I^{-}$. Efficiencies of plating were calculated relative to plating efficiencies on chloramphenicol $(40 \mu \mathrm{g} / \mathrm{ml})$ containing plates for each plasmid except for pRMM61 (40 $\mu \mathrm{g} / \mathrm{ml}$ kanamycin) and pNN392 and pNN393 (100 $\mu \mathrm{g} / \mathrm{ml}$ ampicillin $)$. 
parent transcriptionally dependent phenomenon. The first is that transcription by the strong conI promoter could be topologically altering the rest of the plasmid and that the aadA could produce antisense RNAs that can hybridize to the sense aadA promoter is particularly sensitive to topological perturbations. Second, the antisense transcription of aadA transcripts and interfere with their normal function. Antisense RNA effects have been documented in both eukaryotic (Izant and Weintraub 1984) and prokaryotic systems (Coleman et al. 1984). A third possible mechanism is that of polymerase interference, whereby RNA polymerase molecules transcribing convergently are thought to impede the progress of each other (Ward and Murray 1979). The first mechanism would predict that transcription of sufficient density anywhere on the plasmid should suffice to produce topological perturbations. We tested this possibility by constructing two plasmids containing the tac promoter in the same location, $3^{\prime}$ to the aadA gene but in opposite orientations. Only when tac is transcribing toward the aadA gene, as in pNN390, does the induction of transcription by IPTG interfere with aadA function. This would tend to rule out the first mechanism, assuming no transient localization of the topological perturbations. We can distinguish between the second and third possible mechanisms if the effects are seen in trans because polymerase interference can only function in cis. A test was set up to make a cis/trans determination. A second low-copy-number plasmid bearing an intact aadA gene, pRMM61 (Mulligan and Long 1985), was introduced into a strain already containing the antisense addA plasmid pNN390. No effect of induction of transcription was observed on resistance to spectinomycin. Growth rates at higher spectinomycin levels, which significantly show the growth of pRMM61 alone, were also checked and no difference in growth between pRMM61 alone and pRMM61 plus pNN390 (S.J. Elledge and R.W. Davis, unpubl.) was found. This suggested that the effect was cis. However, we could not control rigorously for equivalent copy numbers of the two plasmids. To exaggerate the antisense phenotype, pNN392 was constructed by placing a tac-aadA construct from pNN390 onto pBR322. Even at this high copy number, an effect of transcription upon spectinomycin resistance was readily observed. This was seen best in a lacI ${ }^{q}$ background, and higher levels of spectinomycin $(1 \mathrm{mg} / \mathrm{ml})$ were needed to observe sensitivity. A mutation was introduced into the aad $A$ gene on this construct by filling in a $B c$ II site in the coding region of aadA to create pNN393. This frameshift mutation completely eliminated any spectinomycin resistance conferred upon the cell by the plasmid. The mutant plasmid was introduced into a strain containing the low-copy-number plasmid pNN388 (Fig. 1) which carried an intact aadA gene. Under these circumstances, it would be expected that pNN393 would be producing massive amounts of nearly perfectly homologous antisense transcripts to $\operatorname{aad} A$ and that if there were antisense interference, we would observe it upon induction of transcription. The results are shown in Table 2. The presence of the plasmid pNN393 made no difference upon the level of spectinomycin resistance whether or not transcription was induced. Therefore, we conclude that the effect we observe occurs only in cis and is due to a form of promoter occlusion. This does not exclude a role for strictly cis antisense RNA effects, i.e., RNA-RNA hybrid formation among transcripts from the same template.

\section{Promoter occlusion has a strong dependence upon promoter strength}

Repression at a distance by convergent transcription may be influenced by a number of factors in vivo, including the distance of separation of promoters, relative promoter strengths, transcription termination and/or transcript stability, and translatability. Many of these factors are poorly understood and are difficult to control properly. However, one factor that can be controlled is the frequency of transcription initiation. To test the effects of varying the level of transcription, the modified conI promoters listed in Figure 2 were placed onto the aadA assay vector pNN388. The pattern of repression was the same for all the conI derivatives conI-1 through conI-16. The typical result for conI-16, pNN395, is listed in Table 2. conI alone interfered with aadA function, as described above, but every other construct bearing the lac operator was able to protect the aadA gene in the absence of IPTG. This means that repressing transcription 15-fold (conI-16 levels divided by two) is enough to interfere with promoter occlusion. Furthermore, the conI-16 construct appeared to have absolutely no phenotypic differences relative to pNN388 in the absence of IPTG.

To define the minimal amount of expression required to interfere with aadA function more precisely, a number of conII constructs were tested that showed lower levels of regulation. These data are shown in Table 3 . The conII, conII-4, and conII-6 promoters were too weak to interfere with aadA function even in a

Table 3. Promoter occlusion dependence on promoter strength

\begin{tabular}{lccc}
\hline \multirow{2}{*}{$\begin{array}{l}\text { Plasmid or } \\
\text { promoter }\end{array}$} & $\begin{array}{l}\text { Fold } \\
\text { repression }\end{array}$ & \multicolumn{2}{c}{$\begin{array}{c}\text { Spectinomycin } \\
\text { resistance }^{\mathrm{b}}\end{array}$} \\
\cline { 3 - 4 } pNN388 & - & $\mathrm{trpR^{- }}$ & $\operatorname{trpR}^{+}$ \\
conII & 0.9 & $\mathrm{R}$ & $\mathrm{R}$ \\
conII-1 & 6.0 & $\mathrm{~S}$ & $\mathrm{R}$ \\
conII-2 & 11 & $\mathrm{~S}$ & $\mathrm{R}$ \\
conII-3 & 14 & $\mathrm{~S}$ & $\mathrm{R}$ \\
conII-4 & 10 & $\mathrm{R}$ & $\mathrm{R}$ \\
conII-5 & 3 & $\mathrm{~S}$ & $\mathrm{R}$ \\
conII-6 & 2 & $\mathrm{R}$ & $\mathrm{R}$ \\
conII-5, pRPG9 & 3.8 & $(\mathrm{R} / \mathrm{S})$ & $(\mathrm{R} / \mathrm{S})$ \\
\hline
\end{tabular}

a The fold repression data is reproduced from Table 1 (ratios; $\mathrm{R}^{-} / \mathrm{R}^{+}$f for clarity.

b (R) Resistance to spectinomycin $(80 \mu \mathrm{g} / \mathrm{ml})$. Under these conditions, the strains plated with an equal efficiency on both chloramphenicol and spectinomycin plates. (S) Sensitivity to spectinomycin, indicating an efficiency of plating of $<10^{-4}$. 
$\operatorname{trp} R^{-}$background. The reason for this is unclear, but they do produce considerably less $\beta$-galactosidase than the other constructs shown in Table 1 . However, conII1, conII-2, conII-3, and conII-5 all showed the ability to interfere with aadA function in a $\operatorname{trp} R^{-}$strain, but conII-1, conII-2, and conII-3 are all resistant to spectinomycin in a $\operatorname{trp} R^{+}$background. This argues that the sixfold repression of conII-1 conferred by the Trp repressor is sufficient to allow full phenotypic expression of aadA. The most interesting construct is conII-5. conII-5 interferes with the phenotype of aadA even in a $\operatorname{trp} R^{+}$background indicating that a threefold reduction in transcription is not sufficient to prevent transcriptional interference. However, introduction of pRPG9 into the strain containing the conII-5 construct produced an intermediate phenotype; microcolonies grew on the spectinomycin plates. Thus, the degree of repression of promoter function needed to prevent promoter occlusion of aadA has been narrowed down; threefold is insufficient, fourfold allows some degree of $a a d A$ phenotypic expression, and sixfold gives full expression. In further support of these numbers, we found that the lac promoter was unable to produce promoter occlusion in our assay. The lac promoter is only three times weaker than tac in vivo (Deuschle et al. 1968b). This shows that promoters that are threefold weaker than tac, either due to repression or to a lower constitutive level of transcription, are insufficiently powerful to inactivate $a a d A$.

\section{Discussion}

Repressors bound at different positions may repress by different mechanisms

Operator sequences have been found at virtually every location in prokaryotic promoters, but the significance of their positioning has remained largely unexplored. We have examined the position effects of two different repressor proteins, the Lac and Trp repressors. The Lac repressor shows both distance and sequence context effects upon its ability to repress and gave a tripartite pattern of repression. The fact that lac operator placement within a given region tended to produce similar levels of repression suggested that the repression in those regions acts through the same mechanism, distinct from mechanisms employed in adjoining regions. We have constructed a model in Figure 5 to summarize our observations.

As mentioned in the introductory section, Straney and Crothers (1987) have shown that the Lac repressor acts by interfering with the formation of the open complex when bound at +1 . It is very likely that the repression plateau we observe for operator positions +5 to +14 works in the same fashion, not by inhibition of RNA polymerase binding, but by interfering with another step such as $\mathrm{RP}_{\mathrm{o}}$ formation, step $\mathrm{B}$ to $\mathrm{C}$ (Fig. 5). Further support for this model stems from the fact that the DNase I footprints for the Lac repressor and RNA polymerase open complex overlap until the operator is located at approximately +17 (Schmnitz and Galas 1979; Spassky et al. 1985; calculated after removing 2 bp from each foot- print to account for the radius of DNase I). Coincidentally, the second repression plateau begins near +17 and extends to +31 . It is likely that the second plateau represents a different mechanism of inhibition because the level of repression is reduced fivefold and formation of $\mathrm{RP}_{\mathrm{o}}$ should not be sterically hindered. The most reasonable explanation is that repressor binding in this region interferes with formation of $\mathrm{RP}_{\mathrm{i}}$, perhaps by influencing the extent or degree of abortive cycling. This is illustrated in Figure 5 as the transition from $\mathrm{C}$ to $\mathrm{D}$. The final plateau consists of only two points, +34 and +40 , that show still lower levels of repression. Carpousis and Gralla (1985) have shown that elongating $R P_{i}$ complexes protect only 10 bases ahead of the next base to be synthesized, i.e., the DNase I footprint extends to +26 when the polymerase is ready to insert base 17 . This means that repressors positioned at +34 and +40 will encounter polymerases that have fully initiated transcription. It is likely, therefore, that repressor molecules at these positions are exerting their influence via the process of transcriptional termination identified by Deuschle et al. (1986a) and illustrated as the transition from $D$ to $E$ in Figure 5. Another explanation for the second plateau is that the process of abortive cycling may be more sensitive to transcription termination than are fully engaged polymerases. The test of these hypotheses will require biochemical analysis.

Besse et al. (1986) performed a similar analysis of operator positioning with lacO. They used the wild-type lac promoter instead of an artificially constructed constitutive promoter. The lac promoter has an additional upstream lac operator sequence directly proceding the capbinding site (Fried and Crothers 1981). Because it has been shown that cooperative interactions can occur between properly spaced operators (Mossing and Record 1986; Kramer et al. 1987), their data may be complicated by periodicity effects caused by the phasing requirements for interaction of closely spaced operators. Although they used a system with a different operator, a different promoter, and different repressor levels, results were obtained that do not show major discrepancies with our results and conclusions.

Operators located within a promoter are more efficient than those positioned near the start of transcription

Repressors bound near the start of transcription repress not by preventing RNA polymerase from binding but by interfering with some other step in promoter activation. Repressors bound farther upstream in promoters at positions overlapping the recognition sequences for RNA polymerase can repress by preventing polymerase binding. We wished to determine which form of repression was intrinsically more efficient. A priori, one might expect that occlusion would be the most efficient. However, the possibility exists that a bound polymerase and repressor may be able to interact to form a stable repressing complex that has a greater stability than either protein alone. We observed that a single trp operator located internally between -19 and +1 was a more effi- 
Figure 5. A model for the operator position effects on repression, i.e., the interference with specific steps in the initiation of transcription. The open rectangles represent promoter DNA. The large ellipse represents RNA polymerase, and the smaller ellipse represents the $\sigma$-subunit. The filled rectangles of the promoter represent regions of DNA that prevent the progression to the next step of transcription initiation (indicated by a letter) when bound by the repressor protein. The filled regions are not meant to represent a footprint of a repressor, but the position where the leftmost edge of an operator must be located to effect repression. The stippled regions are shown to indicate the inexact nature of the boundaries. Boundaries may vary, depending upon both the properties of the promoter and the nature of the repressor protein.

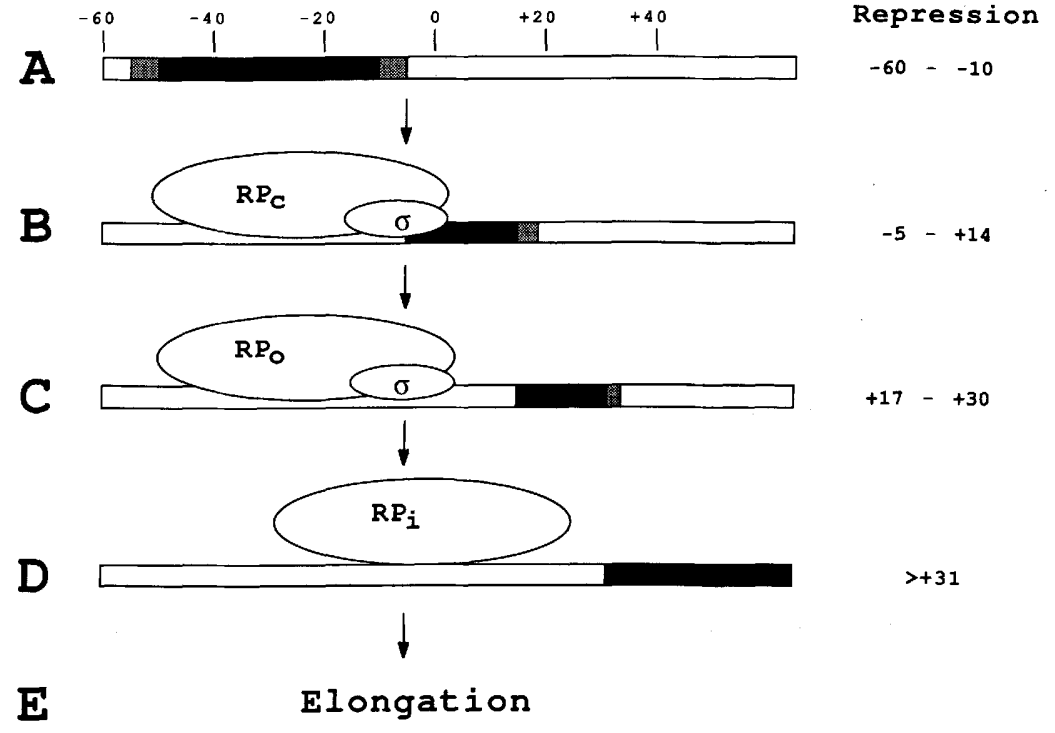

cient repressor in vivo than any trp operator positioned near the start of transcription. A Trp repressor bound in this internal regions is thought to preclude binding of RNA polymerase (Squires et al. 1975). Why do the repressing potentials differ if the operators are identical, having the same affinity for repressor and potentially the same occupancy in vivo? One explanation is the 'polymerase as repressor' model. Once a promoter initiates transcription, the polymerase will cover temporarily the repressor-binding site of operators located near or after the initiation of transcription. A time window will exist in which binding of the repressor is occluded while a second polymerase is free to load and form an open complex. This gives the polymerase a slight temporal advantage for rebinding. However, if the operator is located within the promoter, both repressor and polymerase will have equal access to it once the promoter is cleared. A second explanation is the 'polymerase as bouncer' model. When both polymerase and repressor are bound simultaneously to a promoter, the polymerase can effectively increase the off rate of the repressor, perhaps by melting the DNA in the vicinity of the repressor or by physically bumping it. Both models predict a lower occupancy for operators positioned near the initiation of transcription, the first by decreasing the on rate of the repressor and the second by increasing the off rate. We favor the polymerase as repressor model because increasing the concentration of Trp repressor significantly increases repression of operators near the initiation of transcription, a fact that may be inconsistent with the second model. These models are not mutually exclusive and may function concurrently in vivo. Furthermore, the effectiveness of each mechanism may vary, depending on the position and nature of the repressor and which step in initiation of transcription is being blocked, e.g., B to C or C to D in Figure 5.
Multiple operators in the trp promoter function in vivo

The repression data for trm suggest that at least one of the two exterior operator sites in the trp promoter is occupied in vivo and contributes to repression of the promoter. This work is consistent with the in vitro work of Kumamoto et al. (1987), which suggested that the Trp repressor could bind to four successive major grooves in the $t r p$ promoter. However, the 34-fold repression of trm shows that the majority of the repression is due to the central operator. This explains the observations of Bennett and Yanofsky (1978) that all operator constitutive $\left(\mathrm{O}^{\mathrm{c}}\right)$ mutations isolated in the $\operatorname{trp}$ promoter map to the central operator. Their observations suggest that mutations specifically removing the outer binding sites would not produce a severe regulatory phenotype. Our data concur and demonstrate that the outer two operator sites contribute only fourfold to the regulation of trp. The most severe $\mathrm{O}^{\mathbf{c}}$ mutations isolated by Bennett and Yanofsky would remove two operator sequences simultaneously leaving one exterior operator sequence intact. Coincidentally, the most severe $\mathrm{O}^{c}$ mutations in each half-site of the central operator left a residual twofold repression. An individual twofold repression for the exterior operators would explain precisely the fourfold difference in repression between trp and trm if we assume that the operators act independently. It should be noted that it has not yet been proven that multiple Trp repressors interact with the trp promoter. It is possible but unlikely, given the crystal structure, that one molecule of Trp repressor could interact with all of the sequences important for regulation.

Our data suggests that operator number and positioning can explain the difference in regulation of the trp and $\operatorname{trpR}$ promoters. Operators in the central region of $\mathrm{a}$ promoter, such as found in $t r p$, can confer strong regulation, whereas operators near the start of transcription, 
such as in $\operatorname{trpR}$, confer weaker regulation. Our data do not address the levels of repression of the aroH operon because we have not investigated repression in the -50 to -20 promoter region. Differences in operator affinities among the three promoters appear to be very slight in vitro (Klig et al. 1988) and may only play a significant role when active repressor concentrations approach the $K_{\mathrm{d}}$. Differences in promoter strength may also play a role in precisely defining repression levels. It is possible that weaker promoters are more efficiently repressed by different repression mechanisms that are strong promoters. This question must be addressed in the future to further our understanding of the interaction of repressors and RNA polymerases on the surface of promoter DNA.

\section{Negative regulation at a distance by promoter interference}

We have shown that a strong promoter, conI, can act to silence the aadA gene at a distance. This form of repression occurs only in cis. It shows a directionality dependence and appears to be mediated via transcription. Although general topological interference does not appear to play a role, we cannot rule out a role for transient local superhelicity effects observed during convergent transcription (Wu et al. 1988). We feel that the most likely explanation for this phenomenon is one of transcriptional interference, first described by Ward and Murray (1979), for $\mathrm{pL}$ interference with expression of the trp operon on a $\lambda$ trp-transducing phage. Their work preceded the discovery of antisense RNA effects, so that control was missing from their study. Nevertheless, it is likely that they were observing the same phenomenon. Adhya and Gottesman (1982) also observed promoter interference among promoters transcribing in the same direction. Although similar in principle, it differs mechanistically from our convergent interference because it does not involve RNA polymerase collisions. The problem of polymerase collisions must be a common one in evolution and may be one of the major constraints on the evolution of gene arrangements. Perhaps the cell has evolved special machinery to allow polymerases to bypass one another during transcription. This does not appear to be the case for RNA and DNA polymerases during replication (Brewer 1988), but that involves different circumstances because the DNA replication complex must access both strands of the DNA. Due to its effectiveness as a repression mechanism and the frequency with which it must occur by chance in vivo, one might expect that organisms would exploit this form of repression more frequently than has been observed. In fact, it may be that the effects of convergent transcription have been overlooked in many cases due to the prevailing paradigm that most prokaryotic regulation is exerted through the promoter of a given gene. This is especially true in the cases when the effects are subtle and are coupled with more conventional types of regulation. It is, however, an energetically expensive mechanism of repression and may find selective advantages only when used for limited times such as a short period during a complex developmental program. Cir- cumstances when homeostatic energy considerations are less important, such as during the developmental program of a lytic phage infection, may be another example. It might also find use as a switching mechanism between convergently transcribed genes with antagonistic functions such as must exist among the myriad of regulatory networks controlling gene expression in bacteriophage.

The repressing potential of promoter occlusion is large. It can change sensitivity to spectinomycin over a 100 -fold range (S.J. Elledge and R.W. Davis, unpubl.) when used to repress aadA. We have found a strikingly strong dependence upon promoter strength for this repression. Changes in promoter initiation rates of two- or threefold can have profound effects $\left(10^{5}\right)$ upon plating efficiencies of strains on selective media. The mechanism appears to have the capacity to function as a polymerase density-dependent switch. One possible explanation for this capacity is, at subrepressing transcription rates, RNA polymerase collisions are occurring that slow down the progress of successful transcription from each promoter in a linear fashion. If one promoter becomes sufficiently strong so as to initiate transcription at near maximal rates, it could literally cover the DNA template with polymerase molecules (Kammerer et al. 1986; Brunner and Bujard 1987). Under these conditions, polymerases from the strong promoter could eventually overcome the opposing polymerases by mass action to the point where they began to arrive at and transcribe through the weaker promoter. Once this occurs, the weaker promoter is essentially repressed by the competing polymerases and no longer initiates opposing RNA polymerase molecules, thus enhancing the dominance of the strong promoter and leading to a sigmoidal repression pattern. This model can be broken down into two components: (1) a mutual 'transcriptional interference' due to translocational interference between polymerases, and (2) classical 'promoter occlusion,' where opposing polymerases cover and repress a promoter as described by Adhya and Gottesman (1982). This model is attractive because it explains the strong dependence on promoter strength, as well as requiring the presence of a strong promoter such as tac. Careful measurements of in vivo initiation rates of these promoters while varying the relative promoter strengths will be required to test this and other models. Experiments must also be devised to determine whether opposing RNA polymerase molecules actually interfere with each other during transcription in vivo. They are primarily accessing one strand after initiation. This is a question of fundamental importance.

The in vivo analyses provided in this work have given us insights into the interactions of regulatory molecules on the surface of promoter DNA. The spacing analysis has shown that regulatory molecules can have significant effects when placed much farther away than their normal positions in vivo. This has interesting implications for the pathway of evolution of regulatory sequences in general. The models generated for both local and distant repression will serve to guide future in vitro 
experiments and enhance our understanding of the phenomenon of repression.

\section{Materials and methods}

\section{Bacterial strains}

E. coli JM107 was used as a transformation recipient for all plasmid constructions unless indicated otherwise. JM106 [endA1, gyrA96, thi, hsdR17, supE44, relA1, $\Delta($ lac-proAB)] was used as the $\mathrm{lacI}^{-}$host for measuring unrepressed levels of $\beta$-galactosidase activity. JM107 [endA1, gyrA96, thi, hasR17, supE44, relA1, $\Delta($ lac-proAB $),\left(\mathrm{F}^{\prime}\right.$, traD36, proAB $B^{+}$, lacI $q \mathrm{Z} \Delta \mathrm{M} 15$; Yanisch-Perron et al. 1985) was used as the laclalacZ- host for measuring repressed levels of $\beta$-galactosidase activity. CY 15076 (W3110 tna2, $\Delta l a c \mathrm{U} 169$ ) and CY15075 (W3110 tna2, $\Delta$ lacU169, trpR2) (Kelley and Yanofsky 1985) were used as the $\operatorname{trp} R$ hosts for measuring repressed and unrepressed levels of $\beta$-galactosidase activity. W3110 is a wild-type strain used for measuring Lac repressor titrations. pRPG9 (Gunslaus and Yanofsky 1980) was a gift from M. Kuroda.

\section{Media, enzymes, assays, and genetic methods}

$\beta$-Galactosidase assays were performed as prescribed in Miller (1972). For measurements of the conI derivatives, plasmids in JM106 backgrounds were grown to an $\mathrm{OD}_{600}$ of 1 at $37^{\circ} \mathrm{C}$ in $\mathrm{M} 9$ minimal media supplemented to $40 \mu \mathrm{g} / \mathrm{ml} \mathrm{L}$-proline, $1 \mu \mathrm{l} \mathrm{Bl}$, $0.1 \%$ glucose, and $40 \mathrm{mg} / \mathrm{ml}$ chloramphenicol. Plasmids in JM107 backgrounds were grown under the same conditions, except that the proline was left out of the supplements to select for the $F^{\prime}$. Measurements of $\beta$-galactosidase activity of the $t r p$, trm, and conII derivatives in the CY backgrounds were performed in M9 media supplemented with $0.1 \%$ glucose, 40 $\mu \mathrm{g} / \mathrm{ml}$ chloramphenicol, and $100 \mu \mathrm{g} / \mathrm{ml} \mathrm{L}$-tryptophan.

When drug sensitivities were being measured on plates, LB medium was supplemented with either chloramphenicol (40 $\mu \mathrm{g} / \mathrm{ml})$, spectinomycin $(80 \mu \mathrm{g} / \mathrm{ml})$, kanamycin $(40 \mu \mathrm{g} / \mathrm{ml})$, or ampicillin $(50 \mu \mathrm{g} / \mathrm{ml})$. E. coli is not sensitive to spectinomycin in minimal media. All plates contained an additional $100 \mu \mathrm{g} / \mathrm{ml}$ L-tryptophan. When necessary, IPTG was added to a final concentration of $3 \mathrm{~mm}$.

Restriction endonucleases, $E$. coli DNA polymerase I large fragment, T4 polynucleotide kinase, T4 DNA polymerase, and T4 DNA ligase were purchased from New England Biolabs. Deoxyribonucleotides and ATP were purchased from P-L Biochemicals. Drugs were purchased from Sigma.

\section{DNA sequencing}

Plasmid DNAs were sequenced by the method of Sanger et al. (1977). Single-stranded plasmid DNAs were prepared by the method of Zagursky and Berman (1984), using R408 (Russel et al. 1986) as a helper phage.

\section{Plasmid constructions}

pNN386 was constructed in several steps. First a NotI linker (New England Biolabs) was placed into the PvuII site of pEMBL18 (Dente et al. 1983) that was not in the $l a c Z^{\prime}$ gene to form pEMBLNot. Secondly, a NotI site was inserted into the ClaI site of ptac11 (Amann et al. 1983). The NotI-EcoRI taccontaining fragment was then cloned into NotI-EcoRI digested pEMBLNot to create peEMBLtac. Single-stranded /ss/DNA was prepared from this construct by superinfection with R408 (see above). The ssDNA of pEMBLtac was annealed with an oligo- nucleotide of sequence 5'-CGAGCTCGAATTCCACACATTA- TACGAGCCG-3' and extended with the Klenow fragment of DNA polymerase I and transformed into W3110. This oligonucleotide creates a deletion of the leader region of the tac promoter that removes the lac operator and because high copy number plasmids bearing $1 a c O$ can induce the chromosomal lacZ gene by repressor titration, we screened the transformants on $40 \mu \mathrm{g} \mathrm{X}-\mathrm{Gal} / \mathrm{ml}$ ampicillin plates containing $0.1 \%$ glucose for transformants that remained white. Several transformants were chosen and sequenced by Sanger dideoxy sequencing and a plasmid bearing the proper deletion to form conI (Fig. 2) was named pNN386 (Fig. 1).

pNN388 was constructed in two steps. First, pDPT427 (Taylor and Cohen 1979) a single-copy-number, $\mathrm{Sp}^{\mathrm{r}}, \mathrm{Cm}^{\mathrm{r}}$ plasmid was deleted across its HincII sites to create pSE150. pSE150 has two closely spaced HindIII sites. A HindIII partial was done and a NotI linker inserted to create pNN388 (Fig. 1).

pNN387 was constructed by ligating the $8.2-\mathrm{kb}$ HindIIIBamHI promotorless lacZ fragment from pMC279 (Casadaban et al. 1980) into HindIII-BamHI cut pNN388. Very light blue, $\mathrm{Cm}^{\mathrm{r}}, \mathrm{Sp}^{\mathrm{s}}$ colonies were chosen for analysis. A strain in which the $\operatorname{lac} Z$ gene had replaced the aadA gene was chosen and named pNN387 (Fig. 1).

pNN390 and pNN391 were constructed as follows. First, a portable promoter fragment carrying the Neo gene of Tn5 and ptacll on an EcoRI fragment was obtained from pSE185. This fragment has both the Neo gene and the tac promoter reading in the same direction, with the tac promoter downstream of Neo transcribing into the EcoRI site. We ligated this $1.8-\mathrm{kb} \mathrm{Km}^{\mathrm{r}}$, tac-containing EcoRI fragment made blunt by filling in with the Klenow fragment of DNA polymerase I to NotI-HindIII (filled in by the Klenow fragment of DNA polymerase I), cleaved pNN388, and selected $\mathrm{Cm}^{\mathrm{r}} \mathrm{Km}^{\mathrm{r}}$ colonies. Restriction endonuclease analysis allowed us to distinguish the two possible orientations of inserts. A plasmid with the tac promoter transcribing toward aadA was named pNN390 (Fig. 4). The plasmid with the tac promoter placed in the opposite orientation was named pNN391.

pNN392 was made by ligating the BamHI-HindIII tacaadA-containing fragment from pNN390 into BamHI-HindIIIcleaved $\mathrm{pBR} 322$ and selecting for $\mathrm{Ap}^{\mathrm{r}} \mathrm{Sp}^{\mathrm{r}}$ colonies. This construct gives resistance to high levels of spectinomycin (1 $\mathrm{mg} / \mathrm{ml}$ ) but becomes $\mathrm{Sp}^{\mathrm{s}}$ in the presence of $3 \mathrm{~mm}$ IPTG. pNN393 was made by Klenow filling in the Bcll site in the aadA gene of pNN392.

pNN394 was constructed by ligating the NotI-HindIII fragment containing the conI-1 promoter (see below) into NotIHindIII-cleaved pNN388. pNN395 was the identical construction, only using the conI-16 promoter fragment.

\section{Construction of regulated promoters}

All of the promoters shown in Figure 2 are derivatives of conI. The conI promoter was designed with an EcoRI restriction site placed at the start of transcription followed by a polylinker to facilitate operator positioning. Into the various restriction sites illustrated in Figure 2A on pNN386, we placed an oligonucleotide containing a synthetic lac operator sequence. The lac operator used was the 20-bp symmetrical sequence of Sadler et al. (1983) of sequence 5'-AATTGTGAGCGCGCACAATT-3' (referred to here as $I a C O$. In general, we identified clones by transformation into W3110 and screened for induction of the chromosomal lacZ gene, as described above. lacO-positive 
clones were sequenced as described above and then transferred to pNN387 by ligating the NotI-HindIII con-containing fragments into NotI-HindIII-cleaved pNN387 to assay promoter strength with $\beta$-galactosidase. conI- 1 was made by ligating lacO into EcoRI-cleaved, Klenow filled-in pNN386. conI-2 was made by ligating lacO into SacI-cleaved, T4 DNA polymeraseflushed pNN386. conI-5 was made by ligating lacO into KpnIcleaved, T4 DNA polymerase-flushed pNN386. conI-3 was derived from conI-5 by deleting the internal 4 bp of the SacI site by cleaving with SacI, making flush with T4 DNA polymerase, and ligating. conI-4 was derived from conI-2 by filling in an EcoRI-cleaved conI-2 with the Klenow fragment of DNA polymerase. conI-9 was made by ligating lacO into SmaI-cleaved pNN386. conI- 6 was derived by deleting the internal 4 bp of the SacI site from conI-9 by cleaving with SacI, making flush with T4 DNA polymerase, and ligating. In the T4 reaction, an extra $\mathrm{C}$ at position +11 was deleted from the $\mathrm{SacI}$ site /see sequence in Fig. 2). conI-7 was made by ligating the lacO into Asp718cleaved, Klenow filled-in pNN386. conI-10 was made by ligating the $1 a c \mathrm{O}$ into $\mathrm{XmaI}$-cleaved, Klenow filled-in pNN386. conI-8 was derived from con $I-10$ by deleting the internal 4 bp of the SacI site by cleaving with SacI, making flush with T4 DNA polymerase, and ligating conI-11 was derived from conI-9 by filling in an EcoRI-cleaved conI-9 with the Klenow fragment of DNA polymerase I. conI- 12 was derived from conI- 10 by filling in an EcoRI-cleaved conI-10 with the Klenow fragment of DNA polymerase I. conI-14 was made by ligating the lacO into $\mathrm{XbaI}$ cleaved, Klenow filled-in pNN386. In this construct, a base was deleted from each side of the filled $X b a I$ site. conI -13 was derived by deleting the internal $4 \mathrm{bp}$ of the SacI site from conI-14 by cleaving with SacI, making flush with T4 DNA polymerase, and ligating. conI-15 was derived by filling in an EcoRI-cleaved conI-14 with the Klenow fragment of DNA polymerase I. conI-16 was made by ligating lacO into PstI-cleaved pNN386 made flush by the action of T4 DNA polymerase.

conII was derived from conI by oligonucleotide site-directed mutagenesis. ssDNA was prepared from pNN386 by superinfection with R408 (see above). This ssDNA was annealed with an oligonucleotide of sequence 5 '-ATCCCCGGGTACCATTATACGAGCCG-3' and extended with the Klenow fragment of DNA polymerase I, cleaved with EcoRI, and transformed into JM107. This oligonucleotide creates a deletion of the conI promoter from -5 to +12 , including the EcoRI site. Transformants were sequenced and a clone was chosen with the sequence shown for conII in Figure 3 and designated pNN396. conII-1 was made by ligating the blunt PstI trp operator oligo(trpO) of Figure 3 into KpnI(blunt)-PstI-cleaved pNN396. conII-2 was made by ligating trpO into Asp718(blunt)-PstIcleaved pNN396. conII-3 was made by ligating trpO into SmaI-PstI-cleaved pNN396. conII-4 was made by ligating the trpO oligonucleotide into EcoRI(blunt)-PstI-cleaved pNN386. conII-4 is actually a derivative of conI. conII-5 was made by ligating trpO into BamHI(blunt)-PstI-cleaved pNN396. conII-6 was made by ligating trpO into $X$ baI(blunt)-PstI-cleaved pNN396. All uses of the word blunt after a restriction enzyme site refers to filling in with T4 DNA polymerase. All constructions were sequenced as described above, and their sequences are listed in Figure 3. Each conII promoter derivative was transferred to pNN387 and pNN388 by ligating the NotI-HindIII promoter-containing fragment to NotI-HindIII-cleaved pNN387 or pNN388.

The trp and trm promoters were synthesized as doublestranded oligonucleotides with NotI and HindIII overhangs and cloned directly into NotI-HindIII-cleaved pNN386 for sequencing. They were subsequently subcloned from these sequencing templates onto pNN387 using NotI and HindIII.

\section{Acknowledgments}

We thank T. Baker, M. Kuroda, J. Mulligan, M. Sachs, and C. Yanofsky for critical comments on the manuscript. We also thank M. Kuroda, S. Lewis, and T. Huynh for many helpful discussions. S.J.E. was a Helen Hay Whitney Fellow and an American Cancer Society Senior Fellow during the course of this work. This work was also supported by a NIGMS grant (GM-21891-14) and National Science Foundation grant (DMB-8719440) to R.W.D.

\section{References}

Adhya, S. and M. Gottesman. 1982. Promoter occulsion: Transcription through a promoter may inhibit its activity. Cell 29: 939-944.

Amann, E., J. Brosius, and M. Ptashne. 1983. Vectors bearing a hybrid trp-lac promoter useful for regulated expression of cloned genes in Escherichia coli. Gene 25: 167-178.

Bass, S., P. Sugiono, D.N. Arvidson, R.P. Gunslaus, and P. Youderian. 1987. DNA specificity determinants of Escherichia coli tryptophan repressor binding. Genes Dev. 1: 565-572.

Bennett, G.N. and C. Yanofsky. 1978. Sequence analysis of operator constitutive mutants of the tryptophan operon of Escherichia coli. J. Mol. Biol. 121: 179-192.

Besse, M., B. von Wilcken-Bergmann, and B. Muller-Hill. 1986. Synthetic lac operator mediates repression through lac repression when introduced upstream and downstream from lac promoter. EMBO /. 5: 1377-1381.

Brand, A.H., L. Breeden, J. Abraham, R. Strernglanz, and K. Nasmyth. 1985. Characterization of a 'silencer' in yeast: A DNA sequence with properties opposite to that of a transcriptional enhancer. Cell 41: 41-48.

Brewer, B.J. 1988. When polymerases collide: Replication and the transcriptional organization of the $E$. coli chromosome. Cell 53: 679-686.

Brunner, M. and H. Bujard. 1987. Promoter recognition and promoter strength in the Escherichia coli system. EMBO I. 6: 3139-3144.

Buc, H. and W.R. McClure. 1985. Kinetics of open complex formation between Escherichia coli RNA polymerase and the lac UV5 promoter. Evidence for a sequential mechanism involving three steps. Am. Chem. Soc., 24: 2712-2723.

Carey, J. 1988. Gel retardation at low $\mathrm{pH}$ resolves trp repressorDNA complexes for quantitive study. Proc. Natl. Acad. Sci. 85: 975-979.

Carpousis, A.J. and J. D. Gralla. 1980. Cycling of ribonucleic acid polymerase to produce oligonucleotides during initiation in vitro at the lac UV5 promoter. Biochemistry 19: 3245-3253.

1985. Interaction of RNA polymerase with lac UV5 promoter DNA during mRNA initiation and elongation. Footprinting, methylation, and rifampicin-sensitivity changes accompanying transcriptional initiation. I. Mol. Biol. 183: $165-177$.

Casadaban, M.J., J. Chou, and S.N. Cohen. 1980. In vitro gene fusions that join an enzymatically active $\beta$-galactosidase segment to amino-terminal fragments of exogenous proteins: Escherichia coli plasmid vectors for the detection and cloning of translational initiation signals. I. Bacteriol. 143: 971-980.

Chamberlin, M.E. 1974. The selectivity of transcription. Annu. Rev. Biochem. 43: 721-755.

Coleman, J., P.J. Green, and M. Inouye. 1984. The use of RNAs complementary to specific mRNAs to regulate the expression of individual bacterial genes. Cell 37: 429-436. 
Dente, L., G. Cesareni, and R. Cortese. 1983. pEMBL: A new family of single-stranded plasmids. Nucleic Acids Res. 11: $1645-1651$.

Deuschle, U., G. Reiner, and J. Budard. 1986a. lac repressor blocks transcribing RNA polymerase and terminates transcription. Proc. Natl. Acad. Sci. 83: 4134-4137.

Deuschle, J., W. Kamerer, R. Gentz, and J. Bujard. 1986b. Promoters of Escherichia coli: A hierarchy of in vivo strength indicates alternate structures. EMBO J. 5: 2987-2999.

Downs, D.M. and J. Roth. 1987. A novel P22 prophage in Salmonella typhimurium. Genetics 117: 367-380.

Fried, M. and D.M. Crothers. 1981 Equilibria and kinetics of lac repressor-operator interactions by ployacrylamide gel electrophoresis. Nucleic Acids Res. 9: 6505-6525.

Grove, C.L. and R.P. Gunsalus. 1987. Regulation of the aroH operon of Escherichia coli by the tryptophan repressor. I. Bacteriol. 169: 2158-2164.

Gunsalus, R.P. and C. Yanofsky. 1980. Nucleotide sequence and expression of Escherichia coli trpR, the structural gene for the trp aporepressor. Proc. Natl. Acad. Sci. 77: 71177121.

Hansen, U.M. and W. R. McClure. 1980. role of the $\sigma$ subunit of Escherichia coli RNA polymerase in initiation. J. Biol. Chem. 255: 9564-9570.

Hollingshead, J. and D. Vapnek. 1984. Nucleotide sequence analysis of a gene encoding a streptomycin/spectinomycin adenyltransferase. Plasmid 13: 17-30.

Izant, J.G. and H. Weintraub. 1984. Inhibition of thymidine kinase gene expression by antisense RNA: A molecular approach to genetic analysis. Cell 36: 1007-1015.

Kammerer, W., U. Deuschle, R. Gentz, and J. Bujard. 1986. Functional dissection of Escherichia coli promoters: Information in the transcribed region is involved in late steps of the overall process. EMBO J. 5: 2995-3000.

Kelley, R.L. and C. Yanofsky. 1982. Trp aporepressor production is controlled by autogenous regulation and inefficient translation. Proc. Natl. Acad. Sci. 79: 3120-3124.

1985. Mutational studies with the Trp repressor of Escherichia coli support the helix-turn-helix model of repressor recognition of operator DNA. Proc. Natl. Acad. Sci. 82: $483-487$

Klig, L.S., J. Carey, and C. Yanofsky. 1988. trp repressor interactions with the $t r p$, aroH, and $\iota_{F} R$ operators: Comparison of repressor binding in vitro and repression in vivo. J. Mol. Biol. 202: 769-777.

Klig. L.S., I.P. Crawford, and C. Yanofsky. 1987. Analysis of trp repressor-operator interaction by filter binding. Nucleic Acids Res. 15: 5339-5351.

Kirkegaard, K., H. Buc., A. Spassky, and J.D. Wang. 1983. Mapping of single-stranded regions in duplex DNA at the sequence level: Single-strand specific cytosine methylation in RNA polymerase-promoter complexes. Proc. Natl. Acad. Sci. 80: $2544-2548$.

Kramer, H., M. Niemoller, M. Amouyal, B. Revet, B. von Wilcken-Bergmann, and B. Miller-Hill. 1987. lac repressor forms loops with linear DNA carrying two suitably spaced lac operators. EMBO J. 6: 1481-1491.

Kumamoto, A., W. Miller, and R.P. Gunslaus. 1987. Escherichia coli tryptophan repressor binds multiple sites within the trp and aroH operators. Genes Dev. 1: 556-564.

Majors, J. 1975. Initiation of in vitro mRNA synthesis from the wild-type lac promoter. Proc. Natl. Acad. Sci. 72: 43944398.

Miller, J. 1972. Experiments in molecular genetics. Cold Spring Harbor Laboratory, Cold Spring Harbor, New York.

Mossing, M. and M.T. Record, Jr. 1986. Upstream operators en- hance repression of the lac promoter. Science 233: 889-892. Mulligan, J.T. and S.R. Long. 1985. Induction of Rhizobium meliloti nodC expression by plant exudate is dependent upon nodD. Proc. Natl. Acad. Sci. 82: 6609-6613.

Ptashne, M., A. Jeffery, A.D. Johnson, R. Maurer, B.J. Meyer, C.O. Pabo, T.M. Roberts, and R.T. Sauer. 1980. How the $\lambda$ repressor and cro work. Cell 19: 1-11.

Riggs, A.D., H. Suzuki, and S. Bourgeois. 1969. lac repressor-operator interaction. I. Equilibrium studies. I. Mol. Biol. 48: $67-83$.

Russel, M., S. Kidd, and M.R. Kelley. 1986. An improved filamentous helper phage for generating single-stranded plasmid DNA Gene 45: 333-338.

Sadler, J.R., J. Sasmor, and J.L. Betz. 1983. A perfectly symmetric lac operator binds the lac repressor very tightly. Proc. Natl. Acad. Sci. 80: 6785-6789.

Sanger, F.S., S. Nicklen, and A.R. Coulson. 1977. DNA sequencing with chain-terminating inhibitors. Proc. Natl. Acad. Sci. 74: 5463-5467.

Schmitz, A. and D.J. Galas. 1979. The interaction of RNA polymerase and lac repressor with the lac control region. $\mathrm{Nu}$ cleic Acids Res. 6: 111-136.

Spassky, A., K. Kirkegaard, and J. Buc. 1985. Change in the DNA structure of the lac UV5 promoter during formation of an open complex with Escherichia coli RNA polymerase. Biochemistry 24: 2723-2731.

Squires, C.L., F.D. Lee, and C. Yanofsky. 1975. Interaction of the $\operatorname{trp}$ repressor and RNA polymerase with the $\operatorname{trp}$ operon. I. Mol. Biol. 92: 93-111.

Straney, D.C. and D.M. Crothers. 1985. Intermediates in transcription initiation from the $E$. coli lac UV5 promoter. Cell 43: 449-459.

1987. Lac repressor is a transient gene-activating protein. Cell 51: 699-707.

Taylor, D.P. and S.N. Cohen. 1979. Structural and functional analysis of cloned DNA segments containing the replication and incompatibility regions of a miniplasmid derived from a copy number mutant of NR1. J. Bacteriol. 137: 92-104.

Ward, D.F. and N.E. Murray. 1979. Convergent transcription in bacteriophage lambda: Interference with gene expression. $J$. Mol. Biol. 133: 249-266.

Wang, J.C. and G.J. Giaever. 1988. Action at a distance along a DNA. Science 240: 300-304.

Wu, H-Y., S. Shyy, J.C. Wang, and L.F. Liu. 1988. Transcription generates positively and negatively supercoiled domains in the template. Cell 53: 433-440.

Yannisch-Perron, C.J. Vieira, and J. Messing. 1985. Improved M13 phage cloning vectors and host strains: Nucleotide sequences of the $\mathrm{M} 13 \mathrm{mp} 18$ and pUC19 vectors. Gene 33: $103-119$.

Zagursky, R.J. and M.L. Berman. 1984. Cloning vectors that yield high levels of single-stranded DNA for rapid sequencing. Gene 27: 183-191. 


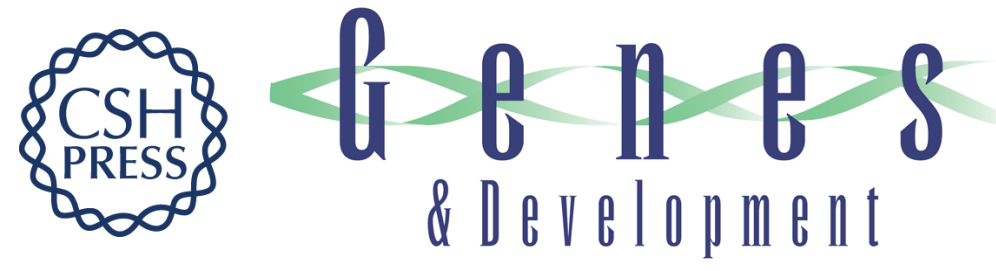

\section{Position and density effects on repression by stationary and mobile DNA-binding proteins.}

S J Elledge and R W Davis

Genes Dev. 1989, 3:

Access the most recent version at doi:10.1101/gad.3.2.185

References This article cites 52 articles, 19 of which can be accessed free at:

http://genesdev.cshlp.org/content/3/2/185.full.html\#ref-list-1

License

Email Alerting

Service

Receive free email alerts when new articles cite this article - sign up in the box at the top right corner of the article or click here.

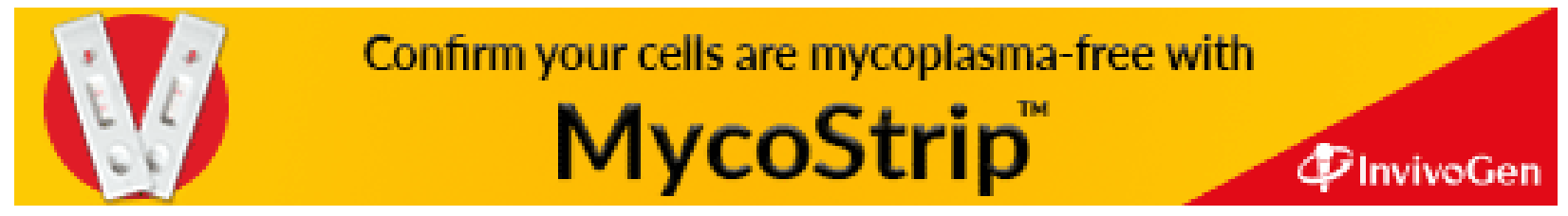

